\title{
Charmonium Spectrum from Quenched Lattice QCD with Tadpole Improvement Action
}

\author{
Da Qing Liu \\ Institute of Theoretical Physics, Chinese Academy of Sciences
}

November 3, 2018

\begin{abstract}
We report here our lattice simulation on the charmonium spectra in the quenched approximation. Because the full adjustment on the nonperturbative parameters such as $C_{E}, C_{B}, m_{0} a_{s}$ and $r_{s}$ needs many calculation time, we only adjust two of them, $m_{0} a_{s}$ and $r_{s}$ ( $\xi_{3}$ plays this role in the paper) but with some rescale for mass splitting. After the rescale, we find that our results are in agreement with the experiment ones.
\end{abstract}




\section{Introduction}

Due to the importance of the charmonium spectra in particle physics, there are many jobs on it in lattice QCD. These jobs did their ways with NRQCD approach ${ }^{[1]}$ or with Wilson action with clover-improvement $[2][3][4][5]$.

We report here our lattice results on the charmonium spectra on anisotropic lattice ( we will also present our results on the bottomonium spectra in the future). Paralleling with references [2]-[5], our results were obtained with the SheikholeslamiWohlert action ${ }^{[6][10]}$ (SW action) as well as with the tadpole improvement. As many authors pointed out, such improvements make us get interesting results with the fewer calculation. In fact, our simulation was done on personal computations.

As pointed out in reference [13], if we adjust parameters to take the right hyperfine splitting in this action, the mass splitting generated by the orbit excitation, including orbit angular momentum and therefore spin orbit interaction, will be incorrect (we call this mass splitting s.o. splitting thereinafter). Therefore, to get the correct charmonium spectra, or the mass splitting, some argument on the mass dependent choice of $C_{E}$ was done in the article [13]. In fact, the choice of the clover coefficient have been performed in various ways, which include applying different orders in perturbation theory ${ }^{[7]}$ and introducing non-perturbative improvement

criteria using Schrödinger function ${ }^{[9]}$. Those schemes, however, need much more computer or calculation time.

If parameters on lattice are not maladjusted so much, then the mass splitting and the gross mass, which is mainly dictated by the static quark mass $M_{1}$, are in different scale. To make the scale at the same, maybe some rescale scheme for the measured mass splitting is also proper. After the rescale, we find that our results are in good agreement with the experiment ones.

The section 2 shows some notations we should considered when we begin to calculate, and the section 3 and 4 is our simulation detail and results. At last, we present the summary in the section 5 .

\section{The Choice of Parameters for Simulation}

To perform the simulation, we adopt the tadpole improvement action for gluon as follows:

$$
S_{G}=\beta\left\{\frac{5}{3} \frac{P_{s p}}{\xi u_{s}^{4}}+\frac{4}{3} \frac{\xi P_{t p}}{u_{s}^{2}}-\frac{1}{12} \frac{P_{s r}}{\xi u_{s}^{6}}-\frac{1}{12} \frac{\xi P_{s t r}}{u_{s}^{4}}\right\}
$$

where $P_{s p}\left(P_{s t}\right)$ is the sum of all spatial(temporal) plaquettes, $P_{s r}$ represents $2 \times 1$ spatial rectangular Wilson loops and $P_{\text {str }}$ is the Wilson loops with 2 spatial and one temporal links. $\xi$ is the aspect ratio:

$$
\xi=\frac{a_{s}}{a_{t}} .
$$


This gluonic action, as many authors pointed out ${ }^{[14]}$, may improve the action up to $O\left(a_{s}^{4}, a_{t}^{2}\right)$.

For quarks, we adopt the SW action. This action was proposed initially to improve the continuum behavior for the fermions. But as suggested by lüscher and Weisz $^{[1]}$, one had better take the scheme with the on-shell improvement. This scheme had been illuminated systematically in reference [13] in action form as well as in Hamilton form. Here we shall use the scheme to determine some parameters in SW action.

We take the SW action as:

$$
\begin{aligned}
S_{q}= & \sum_{x} \bar{\psi}(x)\left\{m_{0} a_{s}+\frac{1}{2}\left[\xi_{1} \gamma_{4} \nabla_{4}+\sum_{i} \gamma_{i} \nabla_{i}\left(1-\frac{1}{6} \triangle_{i}\right)-\xi_{1} \triangle_{4}\right.\right. \\
& \left.\left.-\frac{1}{\xi_{3}} \sum_{i}\left(\triangle_{i}-\frac{1}{12} \triangle_{i}^{2}\right)+C_{E} \sum_{i} \sigma_{4 i} \hat{F}_{4 i}+C_{B} \frac{1}{\xi_{3}} \sum_{i>j} \sigma_{i j} \hat{F}_{i j}\right]\right\} \psi(x), \\
\nabla_{\mu} \psi(x)= & u_{\mu}(x) \psi(x+\hat{\mu})-u_{\mu}^{\dagger}(x-\hat{\mu}) \psi(x-\hat{\mu}), \\
\triangle_{\mu} \psi(x)= & u_{\mu}(x) \psi(x+\hat{\mu})+u_{\mu}^{\dagger}(x-\hat{\mu}) \psi(x-\hat{\mu})-2 \psi(x),
\end{aligned}
$$

where $m_{0}$ is the bare mass of the quark. We shall determine the parameters $\xi_{1}, \xi_{3}$, $C_{E}$ and $C_{B}$ in Eq. (3) in the following.

\subsection{The choice of parameters $\xi_{1}$ and $\xi_{3}$}

The propagator for quark is

$$
<\psi\left(t^{\prime}, p^{\prime}\right) \bar{\psi}(t, p)>=(2 \pi)^{3} \delta\left(p^{\prime}-p\right) C\left(t^{\prime}-t, p\right)
$$

where

$$
C(t, p)=\mathcal{Z}(p) \frac{e^{-E|t|}}{\sinh E}
$$

with the applying of the residue theorem. In Eq. $5, \mathcal{Z}(p)$ is the function which is independent of time $t$. The energy $E$ of the quark with momentum $p$ is determined by

$$
\begin{aligned}
G^{2}+w^{2}+\xi_{1} & =2 G \xi_{1} \cosh E a_{t} \\
G & =m_{0}+\xi_{1}+\frac{1}{\xi_{3}} \sum_{i}\left(1-\cos p_{i}+\frac{1}{6}\left(1-\cos p_{i}\right)^{2}\right) \\
w^{2} & =\frac{1}{9} \sum_{i}\left(\sin p_{i}\left(4-\cos p_{i}\right)\right)^{2} .
\end{aligned}
$$

To get above equation, we set $a_{s}=1$. So, one may expand $G$ and $w^{2}$ according to the power of $p_{i}$ :

$$
\begin{aligned}
G & =m_{0}+\xi_{1}+\sum_{i} \frac{p_{i}^{2}}{2 \xi_{3}}+O\left(p^{6}\right) \\
w^{2} & =\sum_{i} p_{i}^{2}+O\left(p^{6}\right) .
\end{aligned}
$$


At $m_{0}=0$, one gets:

$$
\left(\xi_{1} E a_{t}\right)^{2}+O\left(a_{t}^{4}\right)=\sum_{i} p_{i}^{2}+O\left(p_{i}^{4}\right)
$$

where $a_{t}=1 / \xi$. Because of the Eq. (8), we choose $\xi_{1}=\xi$ with the tuning of the parameter $\xi_{3}$ to make the following dispersion relation of the particle $\psi$ be satisfied:

$$
E^{2}(J / \psi)=m^{2}(J / \psi)+c^{2} \sum_{i} p_{i}^{2}
$$

where $c=1$ is the effective velocity of photon(EVP).

One can not make all the dispersion relations of the calculated particle be satisfied at the same time with the tuning of the sole parameter. Phenomenally, this infraction manifests the fact that the relative movement of two constituent quarks in meson is various with different meson( See appendix for more detail).

\subsection{The Choice of $C_{E}$ and $C_{B}$}

The key points of the choice for the clover coefficient $C_{B}$ is the isospectrum transformation and the redundant coupling. After some algebra calculation, one can easily see that the choice $C_{B}=1$ is proper ${ }^{[13]}$.

There are a few choices of $C_{E}$. For instance, one may choose $C_{E}=1$ or $C_{E}=0$ ${ }^{[12]}$ in NRQCD. In fact, following the method developed in reference [13] and [3], one may see that in this action the parameters should satisfy

$$
\begin{aligned}
C_{B} & =1, \\
\xi_{3} & =\frac{\xi}{1+m_{t}}\left(\frac{1}{\ln \left(1+m_{t}\right)}-\frac{2}{m_{t}\left(2+m_{t}\right)}\right)^{-1}, \\
C_{E} & =\frac{\xi}{\xi_{3}\left(1+m_{t}\right)}+\left(\frac{\xi}{\xi_{3}\left(1+m_{t}\right)}\right)^{2} \frac{m_{t}\left(2+m_{t}\right)}{4},
\end{aligned}
$$

where $m_{t}=\frac{m_{0} a_{s}}{\xi}$. The above condition is at tree level but with tadpole improvement. While for getting the nonperturbative parameters we should adjust these parameters with the same physical principle that led to Eq. (10). For instance, to get the nonperturbative $\xi_{3}$ we adjust $\xi_{3}$ to make Eq. (9) to be satisfied.

We select $C_{B}=1$ for our simulation. While noticing that $C_{E}=\xi / \xi_{3}$ at vanished $m_{t}$ we just take a mass independent setting for $C_{E}$ for simplify:

$$
C_{E}=\frac{\xi}{\xi_{3}} .
$$

The choice in Eq. (10) and (11), however, is at tree level and sometimes a little maladjusted. To compensate the maladjustment will lead to a mass splitting rescale.

Thus we have only two parameters $\xi_{3}$ and $m_{0}$ to be determined in the simulation: one is adjusted to give the correct energy-momentum relation for $J / \psi$ and the other will be rectified to determine the lattice spacing, $a_{s}\left(a_{t}\right)$. 


\section{The Detail for the simulation}

In this work, we calculate five meson masses of charmonium, named as ${ }^{1} S_{0}\left(\eta_{c}\right)$, ${ }^{3} S_{1}(J / \psi),{ }^{1} P_{1}\left(h_{c}\right),{ }^{3} P_{0}\left(\chi_{c 0}\right)$ and ${ }^{3} P_{1}\left(\chi_{c 1}\right)$ and their excited states. The choice of the operator for definite meson is as the standard one ${ }^{[2]}$. These operators are called $\Gamma$ ones in ref. [3].

To determine $\xi_{3}$, we calculate the energy for four low-lying momentum $\frac{L}{2 \pi} \mathbf{p} a_{s}=$ $(0,0,0),(0,0,1),(0,1,1)$ and $(1,1,1)$ of the particle $J / \psi$ and fit them by

$$
\left(E(|\mathbf{p}|) a_{t}\right)=\left(E(0) a_{t}\right)^{2}+\frac{c_{0}^{2}}{\xi^{2}}\left(|\mathbf{p}| a_{s}\right)^{2} .
$$

We tune $\xi_{3}$ to make the relation $c_{0}=1$ be satisfied in one percent accuracy.

There are types of scheme to determine $m_{0}$,or equivalent $a_{t}$. For instance, one may determine $m_{0}$ based on the hyperfine splitting or based on the s.o. splitting. As for the first type, One may take the ratio of the masses of S-state mesons:

$$
\frac{m\left(\psi^{\prime}\right) m\left(\eta_{c}\right)}{m\left(\eta_{c}^{\prime}\right) m(J / \psi)}=0.987 .
$$

And as for the second, one may take, for example, the ratio of the mass of $\chi_{c 1}$ and $J / \psi$

$$
\frac{m\left(\chi_{c 1}\right)}{m(J / \psi)}=1.134
$$

Then, we can extract $a_{t}$ by

$$
a_{t}=\frac{m(1 \bar{S}) a_{t}}{m(1 \bar{S})_{\exp }}
$$

where $m(1 \bar{S})=\left(m\left(1^{1} S\right)+3 m\left(1^{3} S\right)\right) / 4$ is the measured average mass of $1^{1} S$ and $1^{3} S$ state on the lattice and $m(1 \bar{S})_{\exp }$ is the experimental one, 3.0676Gev.

In this paper we iterate $m_{0}$ and $\xi_{3}$ to make both the equation (12) and (13) be hold at the same time.

Because of our computational limitation, we did our simulation on a $L \times T=$ $6^{3} \times 36$ lattice with $\xi=6$.0. Parameters for the simulation are displayed in table 1 .

For a definite $\Gamma$ operator we measure its correlation function as:

$$
C_{\text {state }}(t, \mathbf{p})=\sum_{\mathbf{x}}<\bar{\psi}(\mathbf{x}, t) \Gamma \psi(\mathbf{x}, t) \bar{\psi}(\mathbf{0}, 0) \Gamma \psi(\mathbf{0}, 0)>e^{-i \mathbf{p} \cdot \mathbf{x}}
$$

The energy can be extracted by n-hyperboliccosine ansatz( $n$ is the number of the extracted masses and $\mathrm{T}$ is the periods in the time direction):

$$
C_{\text {state }}(t)=\sum_{j=0}^{n-1} a_{j}\left(e^{-m_{j} t}+e^{-m_{j}(T-t)}\right)
$$


which means that we should find parameters $a(j)$ and $m(j)$ to minimize the function

$$
\sum_{i=l_{i}}^{l_{f}} W_{i}\left(C\left(t_{i}\right)-\sum_{j=0}^{n-1} a_{j}\left(e^{-m_{j} t_{i}}+e^{-m_{j}\left(T-t_{i}\right)}\right)\right)^{2} / C\left(t_{i}\right)^{2},
$$

where $W_{i} \propto \frac{C\left(t_{i}\right)^{2}}{\triangle_{C\left(t_{i}\right)^{2}}}$ is the weight of the correlation $C\left(t_{i}\right)$.

We choose $n=2$ for all the measured particles in the work. Apparently, the excited states we measured here is not the real first excited states in nature but the mixture, the mainly component of which is the first and the second excited states. Thus all the measured excited masses are larger than the experiment ones. We expect that the scheme we adopted in Eq. (14) would decrease this mixing impact.

For each $l_{i}$ and $l_{f}$ in Eq. (18) one may find a group of corresponding parameters $m_{j}$ and $a_{j}$. Then, one may choose a set of parameters $l_{i}, l_{f}, m_{j}$ and $a_{j}$, which make

the function $\sum_{j}\left(\frac{\triangle_{m_{j}}^{2}}{m_{j}^{2}}+\frac{\triangle_{a_{j}}^{2}}{a_{j}^{2}}\right)$ get its minimal points for all the sets. But due to the fact that nearly all the minimal point for a definite operator is approached at the set in which $l_{i}=1$ and $l_{f}=T-1$, we fix our set in which $l_{i}=1$ and $l_{f}=T-1$. This is as expected, for the more measured data means the more information if we excluded the case of $l_{i}=0$ or $l_{f}=T$, in which the counterterms will enter in.

\section{Simulation Results}

In table 2 , we list the results of the typical dispersion relation and the fitting data for low-lying particles at $\beta=3.0$ and $m_{0} a_{s}=1.06$.

From the simulation we know that the EVP for the particle $\eta_{c}$ is equal to the one of the particle $J / \psi$ in about 2 percent. This is obvious from the appendix since their mass splitting is mainly due to the spin splitting ${ }^{[16]}$. The similar result is obtained by other authors ${ }^{[2]-[4]}$.

On the other hand, we find that the EVP in different mesons usually have the relation:

$$
c\left(h_{c}\right) \leq c\left(\chi_{c 1}\right) \leq c\left(\chi_{c 0}\right) \leq c(J / \psi) \approx c\left(\eta_{c}\right) .
$$

This possibly implies that the expanding point $p_{0} \mathrm{~s}$, or roughly saying, the main distribution of the relative momentum, can be estimated as

$$
p_{0}\left(h_{c}\right) \geq p_{0}\left(\chi_{c 1}\right) \geq p_{0}\left(\chi_{c 0}\right) \geq p_{0}(\psi) \approx p_{0}\left(\eta_{c}\right) .
$$

From the data we see that the EVPs for all particles except meson $\eta_{c}$ and $J / \psi$ are usually smaller than unity, which means that the kinetic mass $M_{2}$ is larger than the static mass $M_{1}$ ( see detail in ref. [13]). At the same time, to get the correct $\chi_{J}$ mass splitting, ref. [13] suggested a mass dependence of $C_{E}$, which leads to a scheme of adjustment of three parameters: $m_{0} a_{s}, \xi_{3}$ and $C_{E}$. 
To combine the scale of $M_{1}, M_{2}$ and $M_{E}$ and decrease the number of adjusted parameters, we adopt such progress: we adjust only two parameters $m_{0} a_{s}$ and $\xi_{3}$ but with the mass splitting rescale.

We take Eq. (13) to determine $m_{0} a_{s}$ and measure the mass of particle $\eta_{c}$ and $J / \psi$, but for other particle, since we should consider the s.o. splitting, we redefine its mass as follows.

We rescale the mass splitting between $1^{3} P_{1}$ and averaged $1 S$ state as

$$
m\left(\chi_{c 1}\right)-m(1 \bar{S})=0.443 G e v .
$$

Therefore, the modified mass for a definite meson is

$$
m_{\text {mod }}=0.443 \frac{m a_{t}-m(1 \bar{S}) a_{t}}{m\left(\chi_{c 1}\right) a_{t}-m(1 \bar{S}) a_{t}}+m(1 \bar{S})(G e v),
$$

where $m a_{t}$ is the dimensionless measured mass on the lattice and $a_{t}$ is extracted by Eq. (15).

The simulation parameters are listed in table 3. Our results for scale are not inconsistent with the result obtained from the Sommer scale $r_{0}$ in the calculation of the gluon spectra ${ }^{[17]}$ although they are achieved in very different aspect.

We list our modified charmonium spectra in table 4, which is, as well as in other references, in agreement with the experiment. From the data we find that at the finite lattice spacing the ratio $\frac{m(J / \psi)}{m\left(\eta_{c}\right)}$ is smaller than the experiment one. To get the correct ratio one may decrease $m_{0}$ and therefore achieved a decreased lattice spacing, $a_{s}$. An estimation for the decreased $m_{0}$ shows that it approximately leads to the same result as in reference [17]. To decrease the computer time, we take the scale determined by Eq. (13) with the notice that the ratio between $a_{s}$ obtained by us and that obtained by C.J. Morningstar is nearly the same at different $\beta$, i.e., the ratio is 1.6 at $\beta=3.0$ and 1.7 at $\beta=2.5$.

While compared with our results with those in [5], one may find, that our result for hyperfine splitting is smaller than that in [5]. Maybe the smallness is caused by the too large lattice spacing and/or the contamination of hyperfine splitting by the s.o. terms in the determination of $m_{0} a_{s}$ in Eq. (13).

For the known excited states, our results, as those obtained in ref. [3], are larger than the experiment ones. But for unknown states, it needs some more explore to explain why our result are also larger than those in ref. [3].

While following the reference [13], one should do this rescale between $\chi_{J}$ states. But, from the results we find that our rescale scheme also obtains the correct $\chi_{J}$ states.

\section{Conclusion}

For the simulation of the charmonium spectra, because the ratio between the rest mass $M_{1}$ and the kinetic $M_{2}$ are not always the same, so the scale for mass splitting 
$\Delta m$ and the the gross mass $m$ is different. Since $\Delta m$ is not mainly dictated by the static mass $M_{1}$, reference [13] suggested the following strategy: forget about $M_{1}$ and adjust the bare mass so that the kinetic mass $M_{2}$ takes the physical value.

To make mass splitting and the gross mass at the same scale, one may adjust parameters or rescale mass splitting. This rescale combines the scale of the gross $M$ and the mass splitting, $\Delta M$. After the rescaling, we get the results which are in agreement with the experiment ones despite a little maladjustment of the parameters. 


\section{Appendix A}

From phenomena, one may regard the meson consist of one couple of constituent quark and antiquark and one can also in principle write the wave function for (anti)charm in momentum and spin space. The relative movement between them depicts the different mesons. For instance, the orbital angular momentum in $\eta_{c}$ is S-wave while that in $h_{c}$ is $\mathrm{P}$-wave. On the other hand, for $c \bar{c}$ system the typical velocity in particle is $v^{2} \approx 0.3$, i.e. the energy scales $m_{c} v^{2} / 2$ is about $200-800 \mathrm{Mev}$. This implies that the mass splitting generated by the relative movement between quark and antiquark is mainly dictated by the dynamical behaviour of quarks at the vicinity around $m_{c} v^{2} / 2$. So, maybe the better expanding points in meson is $p_{0}^{2}\left(/ 2 m_{c}\right)$ rather than $p_{0}^{2}=0$, where $p_{0}^{2}$ is phenomenal parameter to describe the relative movement of quarks in mesnon.

In the following we only consider the effect of the relative movement in $1+1$ dimension lattice.

In continuum, the energy-momentum relation for the quark is:

$$
E^{2}(p)=m^{2}+\frac{c^{2} p^{2}}{\xi^{2}}=m^{2}+\frac{c^{2}}{\xi^{2}}\left(p_{0}^{2}+\triangle p^{2}\right)
$$

where all the quantity is dimensionless one, i.e. $E$ is $E a_{t}$, quark mass $m$ is $m a_{t}$ and $p$ is $p a_{s}$. One may rewrite it as:

$$
e^{E}+e^{-E}=e^{m \alpha}+e^{-m \alpha}+\frac{c^{2} \triangle p^{2}}{2 m \alpha \xi^{2}}\left(e^{m \alpha}-e^{-m \alpha}\right)+O\left(\left(\triangle p^{2}\right)^{2}\right),
$$

where $\alpha^{2}=1+\frac{c^{2} p_{0}^{2}}{m^{2} \xi^{2}}=1+\gamma^{2} v_{c}^{2}$. One may redefine the phenomenal parameter $v^{2}$ to set $\gamma=1$.

On the other hand, on an $1+1$ dimension lattice we have:

$$
\begin{aligned}
w^{2} & =w_{0}^{2}+f_{1} \triangle p^{2}, \\
w_{0}^{2} & =\frac{1}{9} \sin ^{2} p_{0}\left(4-\cos p_{0}\right)^{2}, \\
f_{1} & =\frac{\sin p_{0}\left(4-\cos p_{0}\right)}{9 p_{0}}\left(4 \cos p_{0}-\cos 2 p_{0}\right) ;
\end{aligned}
$$

and

$$
\begin{aligned}
G & =G_{0}+f_{2} \triangle p^{2}+O\left(\left(p^{2}\right)^{2}\right) \\
G_{0} & =m_{0}+\xi+\frac{2}{\xi_{3}} \sin ^{2} p_{0} / 2\left(1+\frac{1}{3} \sin ^{2} p_{0} / 2\right) \\
f_{2} & =\frac{\sin p_{0}}{6 \xi_{3} p_{0}}\left(5-2 \cos p_{0}\right)
\end{aligned}
$$

Therefore, according to Eq. (6), on lattice one gets

$$
e^{E}+e^{-E}=\frac{G_{0}}{\xi}+\frac{\xi}{G_{0}}+\frac{w_{0}^{2}}{\xi G_{0}}+\left(\frac{f_{2}}{\xi}+\frac{f_{1}}{\xi G_{0}}-\frac{f_{2}}{G_{0}^{2}}\left(\xi+\frac{w_{0}^{2}}{\xi}\right)\right) \triangle p^{2}+O\left(\left(\triangle p^{2}\right)^{2}\right) .
$$


Comparing with Eq. (ii)and setting $c=1$, one knows that

$$
\begin{aligned}
\xi_{3} & =\frac{1-t^{-2}\left(1+w_{0}^{2} / \xi^{2}\right)}{\frac{\sinh m \alpha}{m \alpha}-\frac{f_{1}}{t}} f_{2} \xi_{3} \xi \\
m_{0} a_{s} & =\xi\left(t-1-\frac{2}{\xi \xi_{3}} g\right)
\end{aligned}
$$

where

$$
\begin{aligned}
g & =\sin ^{2} p_{0} / 2\left(1+\frac{1}{3} \sin ^{2} p_{0} / 2\right) \\
t & =\frac{b+\sqrt{b^{2}-4\left(1+w_{0}^{2} / \xi^{2}\right)}}{2} \\
b & =e^{m \alpha}+e^{-m \alpha}
\end{aligned}
$$

While noticing that functions $f_{2}, f_{1}, G_{0}$ and $w_{0}^{2}$ are even ones for $p_{0}$, one may coarsely replace $p_{0}$ with $\sqrt{p_{0}^{2}}$ in those functions. We expand Eq. (vi) with respect to $m a_{s}$ :

$$
\begin{aligned}
\xi_{3} & \approx\left(1-2 \frac{1+\gamma^{2} v_{c}^{2}}{3} \frac{m a_{s}}{\xi}\right) \xi \\
m_{0} a_{s} & =m a_{s}+O\left(\left(m a_{s}\right)^{3}\right)
\end{aligned}
$$

To applying the above equations, we should consider the intricate additive and multiplicative renormalization ${ }^{[15]}$.

The above argument is somewhat pedagogical, but it manifests the correct qualitative behavior, even on the $3+1$ dimension lattice(see detail in the paper). For instance, from Eq. (viii) it is easily understood that the error of the pole mass will be proportion to $m a$ if we choose $\xi_{3}=\xi$ naively. 


\section{References}

[1] G. P. Lepage et al, Phys. Rev. D46, 4052(1992).

[2] Ping Chen, Phys.Rev. D64, 034509(2001).

[3] M. Okamato et al, Phys.Rev. D65, 094508(2002).

[4] P. Boyle and UKQCD Collaboration, Nucl. Phys. B(Proc.Suppl.), 63(1998).

[5] QCD-TARO Collaboration, Nucl. Phys. Proc. Suppl. 106(2002) 361.

[6] B. Sheikoleslami and R. Wohlert, Nucl. Phys. B259 572(1985).

[7] G. Heatlie et al, Nucl. Phys. B352 266(1001),

M. Luscher and P. Weisz, Nucl. Phys. B479 429(1996).

[8] P. Lepage and P. Machenzie, phys. Rev. D48 2250(1993).

[9] M. Lushcer et al, Nucl. Phys. B491 323(1997),

K. Jansen and R. Sommer, Nucl.Phys. B530 185(1998).

[10] C. Liu and J.P. Ma, Chin. Phys. Lett. 19 640(2002).

[11] M. Lüscher and P. Weisz, Commun. Math. Phys. 97, 59(1985).

[12] G. P. Lepage et al, Phys. Rev. D46, 4052(1992).

[13] A. X. El-Khadra, A. S. Kronfeld and P. B. Mackenzie, Phys. Rev. D55, 3933(1997).

[14] For instance, see C. J. Morningstar and M. Peardon, Phys. Rev. D60, 034509(1999).

[15] M. Luscher et al,Nucl.Phys. B478,365(1996).

[16] S. Godfrey and J. Napolitano, Rev. Mod. Phys. 71, 1411(1999).

[17] C.J. Morningstar and M. Peardon, Phys.Rev. D60, 034509(1999). 


\begin{tabular}{|cccc|}
\hline$\beta$ & $u_{s}^{4}$ & sweep/confg. & confgs. \\
\hline 2.5 & 0.423 & 40 & 100 \\
\hline 2.8 & 0.463 & 40 & 100 \\
\hline 3.0 & 0.486 & 40 & 100 \\
\hline
\end{tabular}

Table. 1 Parameters for the simulation with the scale set by Eq. (13).

\begin{tabular}{|ccccc|}
\hline$\left(\frac{L}{2 \pi}|\mathbf{p}| a_{s}\right)^{2}$ & 0 & 1 & 2. & 3 \\
\hline$\eta_{c}$ & $0.276(3)$ & $0.309(2)$ & $0.340(2)$ & $0.369(2)$ \\
& 0.278 & 0.308 & 0.339 & 0.370 \\
\hline$J / \psi$ & $0.286(1)$ & $0.317(1)$ & $0.349(1)$ & $0.3775(13)$ \\
& 0.286 & 0.317 & 0.348 & 0.3786 \\
\hline$h_{c}$ & $0.409(13)$ & $0.400(10)$ & $0.416(8)$ & $0.432(7)$ \\
& 0.398 & 0.408 & 0.419 & 0.429 \\
\hline$\chi_{c 1}$ & $0.401(9)$ & $0.414(8)$ & $0.432(7)$ & $0.448(7)$ \\
& 0.400 & 0.416 & 0.432 & 0.448 \\
\hline$\chi_{c 0}$ & $0.380(11)$ & $0.405(11)$ & $0.428(12)$ & $0.450(12)$ \\
& 0.380 & 0.404 & 0.427 & 0.451 \\
\hline \hline
\end{tabular}

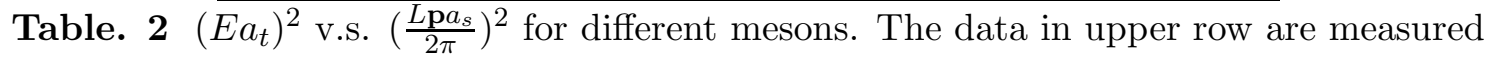
ones and the data in lower row are fitted ones. The fitting parameters $a$ and $b$ of the formula $\left(E a_{t}\right)^{2}=a+b \frac{\left(\mathbf{p} a_{s}\right)^{2}}{\xi^{2}}$ for different meson are $0.2776(20)$ and $1.007(34)$ for $\eta_{c}$, $0.2863(12)$ and 1.010(17) for $J / \psi, 0.398(7)$ and $0.34(11)$ for $h_{c}, 0.3995(58)$ and $0.530(93)$ for $\chi_{c 1}$, and $0.380(8)$ and $0.77(13)$ for $\chi_{c 0}$ respectively. The corresponding velocities of photon for different meson are: 1.003(17) for $\eta_{c}, 1.005(8)$ for $J / \psi, 0.58(10)$ for $h_{c}$, $0.73(6)$ for $\chi_{c 1}$ and $0.88(8)$ for $\chi_{c 0}$ respectively.

\begin{tabular}{|ccccccc|}
\hline$\beta$ & $\xi_{3}$ & $m_{0} a_{s}$ & $a_{s}(f m)$ & $L a_{s}(f m)$ & $\operatorname{EVP}(J / \psi)$ & $\operatorname{EVP}\left(\eta_{c}\right)$ \\
\hline 2.5 & 3.31 & 2.33 & 0.322 & 1.93 & $1.000(10)$ & $1.022(21)$ \\
\hline 2.8 & 3.92 & 1.57 & 0.258 & 1.55 & $1.006(7)$ & $1.018(15)$ \\
\hline 3.0 & 6.38 & 1.06 & 0.208 & 1.25 & $1.005(8)$ & $1.003(17)$ \\
\hline
\end{tabular}

Table. 3 Parameters for the simulation with the scale set by Eq. (13). 


\begin{tabular}{|cccccc|}
\hline \hline state & $\beta=2.5$ & $\beta=2.8$ & $\beta=3.0$ & $a_{s} \rightarrow 0$ & Exp.(Gev) \\
\hline$\eta_{c}$ & $3.030(11)$ & $3.032(11)$ & $3.030(14)$ & $3.030(22)$ & 2.980 \\
\hline$J / \psi$ & $3.080(47)$ & $3.0795(44)$ & $3.080(7)$ & $3.080(10)$ & 3.097 \\
\hline$h_{c}$ & $3.553(135)$ & $3.500(68)$ & $3.539(43)$ & $3.546(117)$ & 3.526 \\
\hline$\chi_{c 1}$ & $3.511(95)$ & $3.511(50)$ & $3.511(32)$ & - & 3.511 \\
\hline$\chi_{c 0}$ & $3.471(139)$ & $3.447(73)$ & $3.436(46)$ & $3.412(119)$ & 3.415 \\
\hline \hline$\eta_{c}^{\prime}$ & $4.309(26)$ & $4.347(17)$ & $3.982(15)$ & $3.766(31)$ & 3.594 \\
\hline$\psi^{\prime}$ & $4.316(11)$ & $4.359(7)$ & $3.993(6)$ & $3.795(13)$ & 3.686 \\
\hline$h_{c}^{\prime}$ & $4.748(107)$ & $4.964(68)$ & $4.633(58)$ & $4.564(126)$ & - \\
\hline$\chi_{c 1}^{\prime}$ & $4.889(92)$ & $5.079(57)$ & $4.689(45)$ & $4.534(102)$ & - \\
\hline$\chi_{c 0}^{\prime}$ & $4.980(150)$ & $5.145(94)$ & $4.721(70)$ & $4.510(161)$ & - \\
\hline \hline
\end{tabular}

Table 4 Results of the charmonium mass $M$ and the mass splitting $\Delta M$ in units of Gev at $\xi=6.0$ with the same scale setting as in Table 1. 